\title{
Recognising the broad array of approaches available for the diagnosis of ancient tuberculosis: Comment on 'Infectious diseases and Neolithic transformations' (Fuchs et al. 2019 The Holocene 29: I545-1557)
}

\author{
David E Minnikin, ' (D) Oona Y-C Lee,' Houdini HT Wu,' \\ Gurdyal S Besra' and Helen D Donoghue ${ }^{2}$
}

\begin{abstract}
The characterisation of ancient tuberculosis is not totally dependent on the recovery of intact genomes. Judicious combinations of ancient DNA fragments and specific lipid biomarkers provide unambiguous diagnosis and these protocols are capable of refinement and extension. Currently, there is no direct evidence for exclusive co-evolution of humans and tuberculosis. A developing body of data suggests that the initial evolution of tuberculosis may readily have taken place in a range of Pleistocene megafauna.
\end{abstract}

\section{Keywords}

ancient tuberculosis, DNA fragment amplification, genomes, lipid biomarkers, paleopathology, Pleistocene megafauna, tuberculosis evolution

Received 26 July 2019; revised manuscript accepted 18 October 2019

The recent interesting study on the distribution of Neolithic tuberculosis (TB; Fuchs et al., 2019) has stimulated a comment on the availability and potential of methods that can be utilised to trace the origins of TB. Several rather pessimistic statements in this paper throw doubt on the practicalities for TB diagnosis in the late Pleistocene and early Holocene. In particular, there is a general statement:

Whether TB was already present before and during the Neolithic or developed much later can only be resolved by genomic data from Neolithic samples ...

Current diagnostic alternatives to full genomics include bone morphology, such as Pott's disease (Figure 5, Fuchs et al., 2019), ancient DNA (aDNA) polymerase chain reaction (PCR)-based fragment amplification and specific profiles of lipid biomarkers (Minnikin et al., 2012, 2015). Subjective bone morphology has great value in suggesting TB infection, but to allow clear TB diagnosis in the absence of genomic data, an integrated combination of aDNA fragment amplification and lipid biomarker analysis has been developed, as comprehensively reviewed by Donoghue et al. (2017) for an invited issue of the journal Diversity.

Fuchs et al. (2019) singled out two particular studies (Hershkovitz et al., 2008; Masson et al., 2015), stating that,

. . . one criticism of the PCR-based method is that it is more prone to contamination and does not allow the differentiation between authentic $M$. tuberculosis and [that of] closely related Mycobacterium species ...
Authoritative, experienced editors and peer reviewers are perfectly capable of dealing with any potential doubts concerning any contamination. Regarding differentiation, authentic Mycobacterium tuberculosis was confirmed by Hershkovitz et al. (2008), who pinpointed the characteristic TbD1 locus with a deletion that is specific for a broadly defined modern lineage of M. tuberculosis (Orgeur and Brosch, 2018). The confirmatory use of TB-specific lipid biomarkers by Hershkovitz et al. (2008) and Masson et al. (2015) is unfortunately disregarded by Fuchs et al. (2019). In the case of a $\sim 9$ ka woman and child from Atlit-Yam, mycolic acid profiles were practically superimposable on those of M. tuberculosis (Figure 1a-e; Hershkovitz et al., 2008; Lee et al., 2015), but the clinching factor was the presence of mycocerosates and mycolipenate (Figure 1f; Lee et al., 2015), the latter being only expressed in authentic members of the M. tuberculosis complex (Jankute et al., 2017). Mycolipenate was also found in a Hungarian $\sim 7 \mathrm{ka}$ individual (HGO-53; Masson et al., 2013) that was included in and discussed by Masson et al. (2015).

Curiously, in an apparent revised opinion, Fuchs et al. (2019) state,

\footnotetext{
'Institute of Microbiology and Infection, School of Biosciences,

University of Birmingham, UK

${ }^{2}$ Centre for Clinical Microbiology, University College London, UK
}

\section{Corresponding author:}

David E Minnikin, Institute of Microbiology and Infection, School of Biosciences, University of Birmingham, Edgbaston, Birmingham BI5 2TT, UK.

Email: d.e.minnikin@bham.ac.uk 

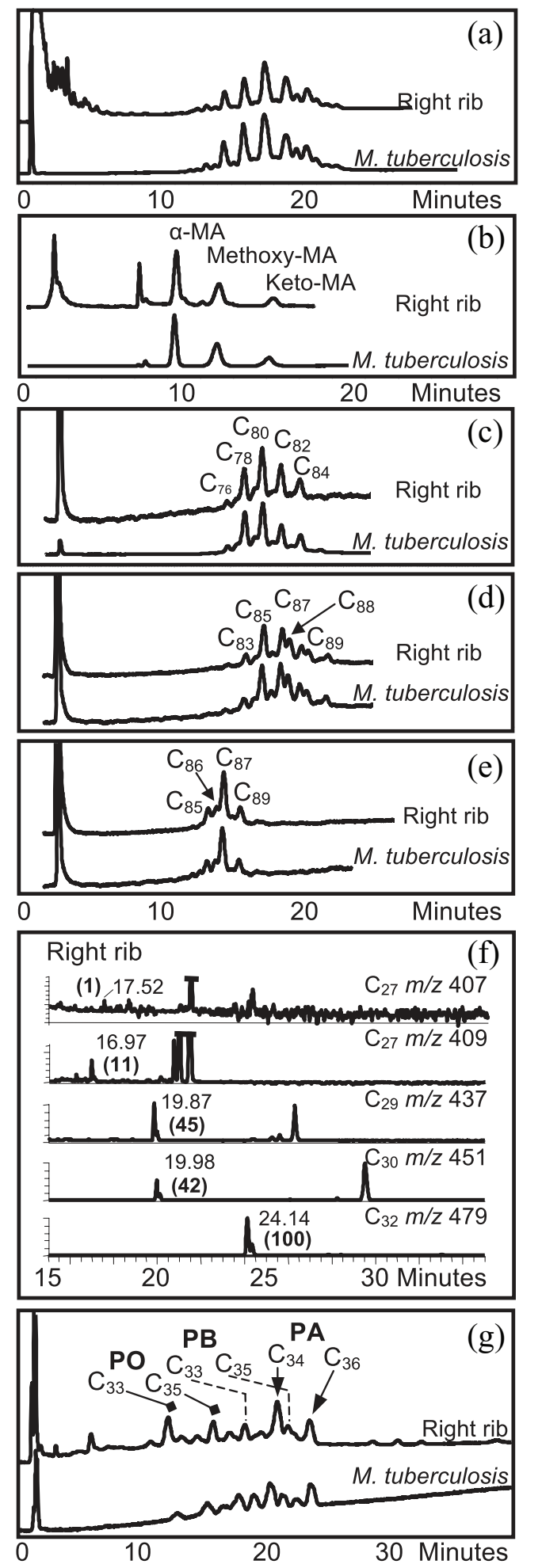

Figure I. Lipid biomarker profiles for the right rib of $\sim 9 \mathrm{ka}$ woman from Atlit-Yam (Donoghue et al., 2017; Lee et al., 2015): (a) total mycolates, reversed-phase HPLC; (b) collected total mycolates (MAs), normal phase; (c) collected $\alpha$-mycolates ( $\alpha-M A s)$, reverse phase; (d) collected methoxymycolates (Methoxy-MAs), reverse phase; (e) collected ketomycolates (Keto-MAs), reverse phase; (f) GC-MS of mycolipenate $\left(C_{27} \mathrm{~m} / \mathrm{z} 407\right)$ and mycocerosates $\left(C_{27}, C_{29}, C_{30}, C_{32}\right)$ characteristic of TB; (g) reversed-phase HPLC of phthiocerol family - PA: phthiocerol A, PB: phthiocerol B, PO: phthiodiolone.

The diagnosis of TB in Neolithic human remains should be based on several lines of evidence, that is, molecular pathogen detection (e.g. by aDNA, lipids) as well as specific and unspecific skeletal symptoms, ideally in the same individual.
To date, these studies have reached their methodological limits (see preceding section; Hershkovitz et al., 2015; Masson et al., 2015 . . .

The mention of 'lipids' is welcome, even though their significance is not indicated. However, it is a hostage to fortune to state categorically that '.. . these studies have reached their methodological limits .... Even if it is not possible to retrieve full genomes, DNA extraction protocols are evolving and improving (Bos et al., 2019; Donoghue et al., 2017). Similarly, the instrumental analysis of lipid biomarkers is not likely to remain static and additional discriminatory lipid classes are available (Jankute et al., 2017). A good example of an additional diagnostic lipid type, ripe for full exploitation, is the TB-specific family of phthiocerols (Figure 1g). Also, other bacterial constituents, such as proteins (Hendy et al., 2016), have untapped potential.

It is essential, when considering the deep origins of $\mathrm{TB}$, to acknowledge the full breadth of available data. One view quoted by Fuchs et al. (2019) is,

... the cause of tuberculosis (TB) is an ancient pathogen that left Africa together with its host $\sim 60,000$ years ago (Comas et al. 2013).

As discussed in depth elsewhere (Donoghue et al., 2017), this opinion is based solely on apparent parallel co-evolution of TB and human mitochondrial genomes with no decisive interconnecting link. The hypothesis of a deep association of TB with Africa arose from the observation that examples of most modern TB clades can be found on the continent and a likely ancestral taxon, 'Mycobacterium canettii', has particular links with the 'Horn of Africa' (Orgeur and Brosch, 2018). However, to date, no direct evidence for any TB in Africa, before the Holocene, has been recorded.

An alternative scenario for TB evolution, quoted by Fuchs et al. (2019), is that,

... two ancient genome studies suggest estimates of fewer than 6,000 years for the emergence of the most recent common ancestor of the bacterium (Bos et al. 2014; Kay et al. 2015).

As it stands, this statement is perfectly valid, but care must be taken to avoid speculative over-interpretation leading to suggestions that cases before $\sim 6 \mathrm{ka}$ are not authentic TB (Bos et al., 2014, 2019). The onset of Neolithic epidemiological and demographic changes, outlined by Fuchs et al. (2019), may have accelerated TB transmission and evolution in the Bronze Age resulting in modern clades that are only traceable back to $\sim 6 \mathrm{ka} \mathrm{BP}$.

However, Fuchs et al. (2019) have chosen to disregard a viable hypothesis for TB evolution before the Holocene, which involves co-evolution with Pleistocene megafauna (Donoghue et al., 2017; Jankute et al., 2017; Minnikin et al., 2015). This scenario is founded on the landmark case of a $\sim 17 \mathrm{ka}$ bison metacarpal with TB-indicative lesions that were confirmed by aDNA fragment amplification (Rothschild et al. 2001) and pristine lipid biomarkers, including TB-definitive mycolipenate (Lee et al., 2012, 2015). As correctly noted by Fuchs et al. (2019), infectious agents require sufficient population numbers to survive and humans were thinly spread in the Pleistocene. Consequently, considering the widespread Northern Hemisphere distribution of TB-diagnostic lesions in bison, mastodons and other megafauna, it is very plausible to engage with a Pleistocene zoonotic origin for TB (Donoghue et al., 2017; Jankute et al., 2017; Minnikin et al., 2015). Briefly, a general picture can be envisaged as involving initial TB evolution, in hordes of megafauna, from environmental mycobacteria to ancestors related to low-pathogenicity organisms related to ' $M$. canettii' (Minnikin et al., 2015). A decisive increase in hydrophobicity, 
consequent aerosol transmission and bottleneck refinement would lead to modern TB clades infecting humans and animals in the late Pleistocene/early Holocene (Jankute et al., 2017). Increasing evidence for multiple human African exit and re-entry would explain the variety of TB clades in Africa. Indeed, the concentration of ' $M$. canettii' in the Horn of Africa (Orgeur and Brosch, 2018) may be simply a result of this being on one of the favoured traffic routes.

The principal aim of this Comment is to highlight awareness of various positive optimistic approaches for the diagnosis of ancient TB. In addition, it is important to maintain a realistic focus on scenarios for TB origins that are supported by solid indisputable published biomarker data (Donoghue et al., 2017).

\section{Funding}

The author(s) received no financial support for the research, authorship and/or publication of this article.

\section{ORCID iD}

David E Minnikin iD https://orcid.org/0000-0002-9486-8999

\section{References}

Bos KI, Harkins KM, Herbig A et al. (2014) Pre-Columbian Mycobacterium genomes reveal seals as a source of New World human tuberculosis. Nature 514: 494-497.

Bos KI, Kühnert D, Herbig A et al. (2019) Paleomicrobiology: Diagnosis and evolution of ancient pathogens. Annual Review of Microbiology 73: 639-666.

Comas I, Coscolla M, Luo T et al. (2013) Out-of-Africa migration and Neolithic co-expansion of Mycobacterium tuberculosis with modern humans. Nature Genetics 45: 1176-1182.

Donoghue HD, Taylor GM, Stewart GR et al. (2017) Positive diagnosis of ancient leprosy and tuberculosis using ancient DNA and lipid biomarkers. Diversity 9: 46.

Fuchs K, Rinne C, Drummer C et al. (2019) Infectious diseases and Neolithic transformations: Evaluating biological and archaeological proxies in the German loess zone between 5,500 and 2,500 BCE. The Holocene 29: 1545-1557.

Hendy J, Collins M, Teoh KY et al. (2016) The challenge of identifying tuberculosis proteins in archaeological tissues. Journal of Archaeological Science 66: 146-153.
Hershkovitz I, Donoghue HD, Minnikin DE et al. (2008) Detection and molecular characterization of 9,000-year-old Mycobacterium tuberculosis from a Neolithic settlement in the Eastern Mediterranean. PLOS ONE 3: e3426.

Jankute M, Nataraj V, Lee OY-C et al. (2017) The role of hydrophobicity in tuberculosis evolution and pathogenicity. Scientific Reports 7: 1315.

Kay GL, Sergeant MJ, Zhou Z et al. (2015) Eighteenth-century genomes show that mixed infections were common at time of peak tuberculosis in Europe. Nature Communications 6: 6717.

Lee OY-C, Wu HHT, Besra GS et al. (2015) Lipid biomarkers provide evolutionary signposts for the oldest known cases of tuberculosis. Tuberculosis 95(Suppl. 1): S127-S132.

Lee OY-C, Wu HHT, Donoghue HD et al. (2012) Mycobacterium tuberculosis complex lipid virulence factors preserved in the 17,000-year-old skeleton of an extinct bison, Bison antiquus. PLoS ONE 7: e41923.

Masson M, Bereczki Z, Molnár E et al. (2015) 7,000 year-old tuberculosis cases from Hungary: Osteological and biomolecular evidence. Tuberculosis 95(Suppl. 1): S13-S17.

Masson M, Molnár E, Donoghue HD et al. (2013) Osteological and biomolecular evidence of a 7,000-year-old case of hypertrophic pulmonary osteopathy secondary to tuberculosis from Neolithic Hungary. PLoS ONE 8: e78252.

Minnikin DE, Lee OY-C, Wu HHT et al. (2012) Molecular biomarkers for ancient tuberculosis. In: Cardona P-J (ed.) Understanding Tuberculosis: Deciphering the Secret Life of the Bacilli. Rijeka, Croatia: InTech - Open Access Publisher, pp. 1-36. Available at: http://www.intechopen.com/books/ understanding-tuberculosis-deciphering-the-secret-life-ofthe-bacilli.

Minnikin DE, Lee OY-C, Wu HHT et al. (2015) Ancient mycobacterial lipids: Key reference biomarkers in charting the evolution of tuberculosis. Tuberculosis 95(Suppl. 1): S133-S139.

Orgeur M and Brosch R (2018) Evolution of virulence in the Mycobacterium tuberculosis complex. Current Opinion in Microbiology 41: 68-75.

Rothschild BM, Martin LD, Lev G et al. (2001) Mycobacterium tuberculosis complex DNA from an extinct bison dated 17,000 years before the present. Clinical Infectious Diseases 33: 305-311. 